\title{
Association of physical performance tests with frailty indicators and oxidative stress markers in a sample of a community-dwelling elderly population.
}

\author{
Levy Munguia', Vannia Solis', Eduardo Meaney ${ }^{1}$, Israel Ramirez-Sanchez ${ }^{1}$, Francisco Villarreal ${ }^{2}$, \\ Javier Perez-Duran ${ }^{1}$, Ivan Rubio-Gayosso ${ }^{1}$, Alicia Ortiz ${ }^{3}$, Guillermo Ceballos ${ }^{1 \#, ~ N a y e l l i ~ N a j e r a ~}{ }^{\text {*\# }}$ \\ ${ }^{1}$ Escuela Superior de Medicina, Instituto Politecnico Nacional, Mexico \\ ${ }^{2}$ School of Medicine, UCSD, USA \\ ${ }^{3}$ Escuela Nacional de Ciencias Biologicas, Instituto Politecnico Nacional, Mexico \\ \#These authors have equally contributed to this study.
}

\begin{abstract}
Aim: Frailty is a syndrome that commonly affects the elderly population and is characterized by physical, mental and metabolic decline, increasing the risk for disability and death. The aim of this study was to study the relationship amongst the performance in 6 min walk distance, 2-min step, sit-up, and up and go tests with frailty and its relationship with select, pre-identified factors in a communitydwelling elderly Mexican population.

Methods: The study comprised 170 individuals between 65-90 y old from 5 community centres in Mexico City. Frailty was defined using the Fried Frailty Phenotype (FFP) criteria. A set of variables were used to explore associations between mobility and frailty, health conditions, and quality of life.

Results: Mean age of participants was $72.6 \pm 6.5 \mathrm{y}, 82.3 \%$ of which were women. The prevalence of frailty was $25.9 \%(n=44)$. Frailty was more frequent in women, in persons aged $80 \mathrm{y}$ and over as well as in those demonstrating low performance in the 2-min step, sit-up, and up and go tests. Lipoperoxidation measured as malondialdehyde (MDA) and carbonyl blood concentrations, as markers of oxidative stress, were significantly elevated in frail vs. non-frail subjects

Conclusion: Frailty is a common pathology in a sample of the elderly community in Mexico. It is associated to low physical performance, quality of life, body composition and metabolic parameters and high oxidative stress.
\end{abstract}

Keywords: Elderly, Frailty, Fried frailty phenotype, Oxidative stress, Physical performance.

Accepted on September 18, 2018

\section{Introduction}

The demographic segment of subjects aged $65 \mathrm{y}$ old or more in Mexico represents currently $9 \%$ of the country's population. This proportion has increased rapidly over the last few decades, as a result of both; a decrease in birth rate and a longer life expectancy [1]. Ageing is frequently associated with the development of multiple morbid conditions as; decreased physical and mental abilities, dependence and low quality of life. Age-related physical and mental deficiencies constitute a heavy burden for the elders, their families and the public health care systems [2]. The expanded incidence and prevalence of frailty, due to the demographic ageing of the population is a major concern everywhere [3].

Although the concept has evolved, the most accepted definition of frailty is the one proposed by Fried [4] that comprises five criteria: weight loss without an intentional weight-losing regime, weakness, exhaustion or fatigue, slow walking speed and low physical activity. Frailty leads to an increased risk for falls, disability, hospitalizations and mortality [4]. The prevalence of frailty in Latin America and the Caribbean ranges from 7.7\%-42.6\% [5]. In Mexico, several studies report prevalence of $14.1 \%$ to $37.2 \%$, ranking among the continent's highest [6].

Numerous studies have described the association of decrease of both handgrip strength and physical performance tests, with functional disability and mortality [7-9]. The use of specific tests for physical capacity evaluation in elderly individuals is helpful to determine which patients are most at risk of accelerated multi-organ decline [10]. According to the 2012 National Health and Nutrition Survey, $26.9 \%$ of the elderly have some degree of functional limitation and despite the 
government's efforts to include older people in social programs; $\sim 40 \%$ do not receive medical attention [11].

Recent evidence demonstrates that oxidative stress plays an important role in activating signaling pathways involved in muscle wasting and loss of metabolic resiliency, which may contribute to frailty [12]. It has been shown that reactive oxygen species levels among the elderly are increased, leading to oxidative damage to biomolecules including carbohydrates, lipid, proteins and DNA [13].

The aim of this study was to establish the association of physical mobility, frailty, cardiovascular risk biomarkers and oxidative stress markers in a sample of community-dwelling elders' population in Mexico City.

\section{Methods}

The study was conducted between October and December 2016. An invitation to participate was posted in five community centres located in Mexico City. The inclusion criteria were: community-dwelling healthy subjects over the age of $65 \mathrm{y}$ that were mobile (capable of move without aid). Non-inclusion criteria include; habitual consumption of antioxidant supplements, benzodiazepines, protein supplements and active chronic infections or malignancies. Volunteers signed informed consent indicating their agreement to participate including permit to publish the obtained results. The ethics and research committee of Escuela Superior de Medicina of the Instituto Politecnico Nacional approved this study. The following data were collected for demographic purposes and grouped in categories: anthropometry, medical history and functional assessment.

\section{Study variables}

Classification of frailty: We considered the 5-criteria proposed by Fried frailty criteria (FFP) that defines frailty by the presence of three or more of the following: unintentional weight loss, weakness, self-reported exhaustion or fatigue, slow walking speed and low physical activity. Slowness was considered if the individual was in the lower $20 \%$ of its group for sex, based on the time to perform the up and go test. Weakness was defined as maximum grip strength of $\leq 30 \mathrm{~kg}$ for men and $\leq 20 \mathrm{~kg}$ for women. Exhaustion/fatigue was considered if the subject experienced severe tiredness. Low physical activity was defined when the subject did not perform regular exercise. Weight loss was diagnosed when the subject reported unintentional weight loss of $5 \mathrm{~kg}$ or more in the previous $2 \mathrm{y}$.

\section{Anthropometric measures}

Anthropometric variables were body weight, body mass index (BMI), and waist circumference and body composition. Measures were obtained with the subject barefooted and lightly dressed. Height was measured with a wall-stadiometer. Body muscle mass, visceral fat mass and body fat percent were obtained using a bioelectrical impedance method by Omron HBF-516 apparatus. The abdominal circumference (waist) was measured with a fiber-glass metric tape, at the mid-point between the top of the hip bone and the lowest rib of each subject at the end of expiration.

\section{Functional assessment}

Six-minute walk test: Subjects were instructed to walk at their normal gait pace during $6 \mathrm{~min}$, in a straight indoor corridor of $30 \mathrm{~m}$ of length, with a flat and hard surface covered by squared tiles measuring $33 \times 33 \mathrm{~cm}$. The estimation of the walked distance was done taking into account those markers and using a flexible retracting tape measure to include the inter-tile distance.

Two-minute step in place test: Subjects in a standing up straight position, started marching in place during two minutes, raising each knee as many times as possible.

Sit-up test: Subjects go up and sit down as quickly as possible during a $30 \mathrm{~s}$ period, keeping their arms folded across the chest.

Up and go test: Subjects seated back in an arm chair, on the word "go" they got up and walked through a 3-meter line on the floor, turned around and walked back to the chair and sat down. Time was recorded by stopwatch.

\section{Handgrip strength}

Handgrip strength was determined using a handgrip dynamometer, measuring the intensity of three consecutive grips done with the dominant hand. The highest value was considered as the patient's muscle strength.

\section{Metabolic status}

After a fasting phase of $12 \mathrm{~h}$, a venous blood sample was taken to determine glycemia and a lipid profile (total cholesterol, triglycerides (TG), cholesterol of high-density lipoproteins (HDL-c) and cholesterol of low-density lipoproteins (LDL-c)). The index TG/HDL reflecting coronary risk [14] and insulin resistance [15] was also estimated.

\section{Oxidative stress biomarkers}

Lipid oxidation was measured using the malondialdehyde (MDA) assay. In this reaction, a lipid peroxidation product is produced with 1-methyl-2-phenylindole which in the presence of MDA produces a color detected spectrophotometrically at $586 \mathrm{~nm}$. Protein carbonylation was measured using an assay for detection of carbonyl groups that relies on 2, 4dinitrophenylhydrazine as a substrate. The reaction leads to the formation of a stable 2, 4-dinitrophenyl hydrazone product, which is evaluated spectrophotometrically at $375 \mathrm{~nm}$.

\section{Quality of life}

For the assessment of daily activities, performance and quality of life perception, we used the EuroQuality of Life 5D instrument (EQ-5D) that consists of a visual analog scale (VAS) and a descriptive system covering 5 items (mobility, 
Association of physical performance tests with frailty indicators and oxidative stress markers in a sample of a community-dwelling elderly population

self-care, usual activity, pain/discomfort and anxiety/ depression) in a $0-100$ scale [16].

\section{Statistical analyses}

Data are reported as mean \pm SD. Analysis was performed with Graph Pad Prism V7.0 and SPSS V24. Statistical significance was considered when $p$-value $<0.05$. To assess the association of anthropometric, medical and performance factors with frailty, a bivariate analysis was performed using a Fisher's test for categorical variables. A Student's t-test was used for continuous variables stratified by sex. Adjusted multiple logistic regression models were fitted including all the covariates (age, sex, marital status, years in school, smoking status, number of medications and chronic diseases and performance tests) reporting odds ratio (OR), 95\% confidence intervals (CI). Analysis of co-variances (MANCOVA) was used as necessary, controlling for confounders. All data analysis was approved by an expert statistician.

\section{Results}

Analysis included 170 participants aged 65 y and over from a total of 900 members of five communities centres in Mexico City. The distribution of general variables is shown in Table S1. Mean age was $72.6 \pm 6.5$ (range: $65-90$ ), $82.3 \%$ of whom were women $(140 / 170)$.

The majority of the study participants were overweight (71.1\%), with increased waist circumference $(81.2 \%)$ and elevated body fat percentage (77\%). Regarding the physical performance battery test, there were no significant differences between genders. The mean results (Table S2) of these tests were as follows: In the 6 min walk test (381.8 \pm 82.1 meters), in the 2 min step in place test (159.2 \pm 35.3 repetitions), in the sit-up test (13.8 \pm 3.4 repetitions), in the up and go test ( $8.6 \pm$ 2.2 seconds) and handgrip strength $(17.2 \pm 4.1 \mathrm{~kg})$.

More men reported moderate problems than women in mobility (Table S3) $(40 \%$ vs. $11.4 \%, \mathrm{P}=0.0005)$. Men reported more problems $v s$. women in the usual activities category $(40 \%$ vs. $14.3 \%, \mathrm{P}=0.003$ ), and also more moderate pain/discomfort vs. women $(20 \%$ vs. $6.4 \%, \mathrm{P}=0.02)$. Men reported more anxiety $v s$. women $(60 \%$ vs. $14.3 \%, \mathrm{P}<0.0001)$. However test results showed a better quality of life score in men (78vs. 76, $\mathrm{P}=0.048$ ).

\section{Frailty}

The most common component of the FFP found in women (Table S4) was exhaustion with $55.7 \%(\mathrm{n}=78)$, while in men it was low physical activity, with a frequency of $33.3 \%(n=10)$. The prevalence of FFP was $25.9 \%(\mathrm{n}=44)$, without difference between genders $(27.9 \%$ women $v$ s. $16.7 \%$ men, $\mathrm{P}=0.25)$. The peak of prevalence of frailty and its components (Figure 1A) is found from the age of $81 \mathrm{y}$, with the most affected categories being weight loss, slowness and low physical performance.

Table 1. Metabolic variables.

\begin{tabular}{llll}
\hline \multirow{2}{*}{ Variable } & No frail & Frail & p value \\
\cline { 2 - 4 } & $\mathbf{n = 1 2 6}$ & $\mathbf{n = 4 4}$ & \\
\hline Glucose $(\mathrm{mg} / \mathrm{dL})$ & $98.8 \pm 10.1$ & $95 \pm 14.3$ & 0.12 \\
\hline $\mathrm{TC}(\mathrm{mg} / \mathrm{dL})$ & $200.6 \pm 32.5$ & $190.6 \pm 30.9$ & 0.44 \\
\hline TG $(\mathrm{mg} / \mathrm{dL})$ & $155.8 \pm 18.3$ & $153.7 \pm 18.4$ & 0.07 \\
\hline C-HDL $(\mathrm{mg} / \mathrm{dL})$ & $42.1 \pm 4.1$ & $43.4 \pm 6.5$ & 0.08 \\
\hline C-LDL $(\mathrm{mg} / \mathrm{dL})$ & $127.9 \pm 35.3$ & $121 \pm 32.9$ & 0.36 \\
\hline TG/HDL $(\mathrm{Index})$ & $4.6 \pm 1.2$ & $3.9 \pm 1.1$ & 0.13 \\
\hline Lipid peroxidation $(\mathrm{nm} / \mathrm{ml})$ & $8.3 \pm 0.1$ & $9.1 \pm 0.3$ & 0.01 \\
\hline Protein carbonyls $(\mathrm{nm} / \mathrm{ml})$ & $59.3 \pm 0.6$ & $64.5 \pm 0.8$ & $<0.0001$ \\
\hline
\end{tabular}

A

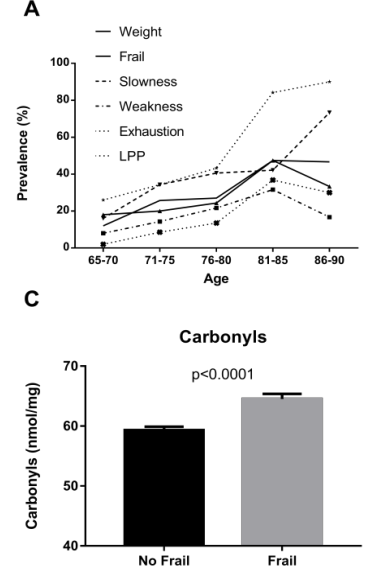

B

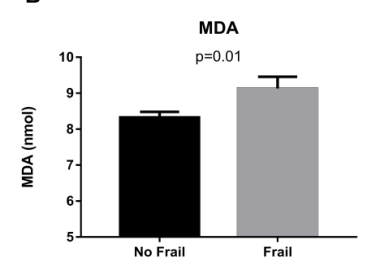

Figure 1. (A) Prevalence of Fried frailty phenotype (FFP) criteria (\% of population ages 65-90) in a sample of elder Mexican population. Data shows an age-dependent increase in slowness, weakness and exhaustion resulting in an increase in frailty; (B) Lipid peroxidation plasma levels measured using the malondialdehyde (MDA) assay. There is a statistical difference $(p=0.01)$ in MDA concentration between subjects with frailty and those no frail. Data are expressed as mean \pm SEM. Statistical differences were determined using " $t$ "test; (C) Protein carbonylation plasma levels measured using 2, 4dinitrophenylhydrazine (DNPH) as a substrate. There is a statistical difference $(p=0.0001)$ in carbonyls concentration between subjects with frailty and those no frail. Data are expressed as mean $\pm S E M$. Statistical differences were determined using " $t$ "-test.

Table 1 shows blood based metabolic parameters including; high-density lipoprotein cholesterol (HDL-c), low-density lipoprotein cholesterol (LDL-c), total cholesterol and triglycerides, as well as glucose concentration. No association was found between frailty and metabolic profile. However, frail subjects had higher plasma lipid peroxidation $v s$. non-frail subjects $(\mathrm{P}<0.01)$ (Figure 1B). As noted in Table 2, higher lipid peroxidation levels were associated with increased odds ratios (OR) of frailty, for men (OR 4.89, 95\% CI 0.61-31.3) and for women (OR 3.5, 95\% CI 1.53-8.5). Frail subjects also had higher levels of protein carbonylation vs. non-frail subjects $(\mathrm{P}<0.0001)$ (Figure 1C). Higher carbonylation levels were associated with increased odds of frailty, for men (OR 4.3, 
95\% CI 0.52-56.5) and for women (OR 3.05, 95\% CI 1.44-6.6) (Table 2).

Univariate analysis shows in women, an association between frailty and age 80 and over (Table 2), under multiple medications, affected by several comorbidities and increased waist circumference. For men, frailty was associated to age over 80 and to a low education level and increased waist circumference.

Table 2. Univariate associations between frailty and the clinical, anthropometric, characteristics.

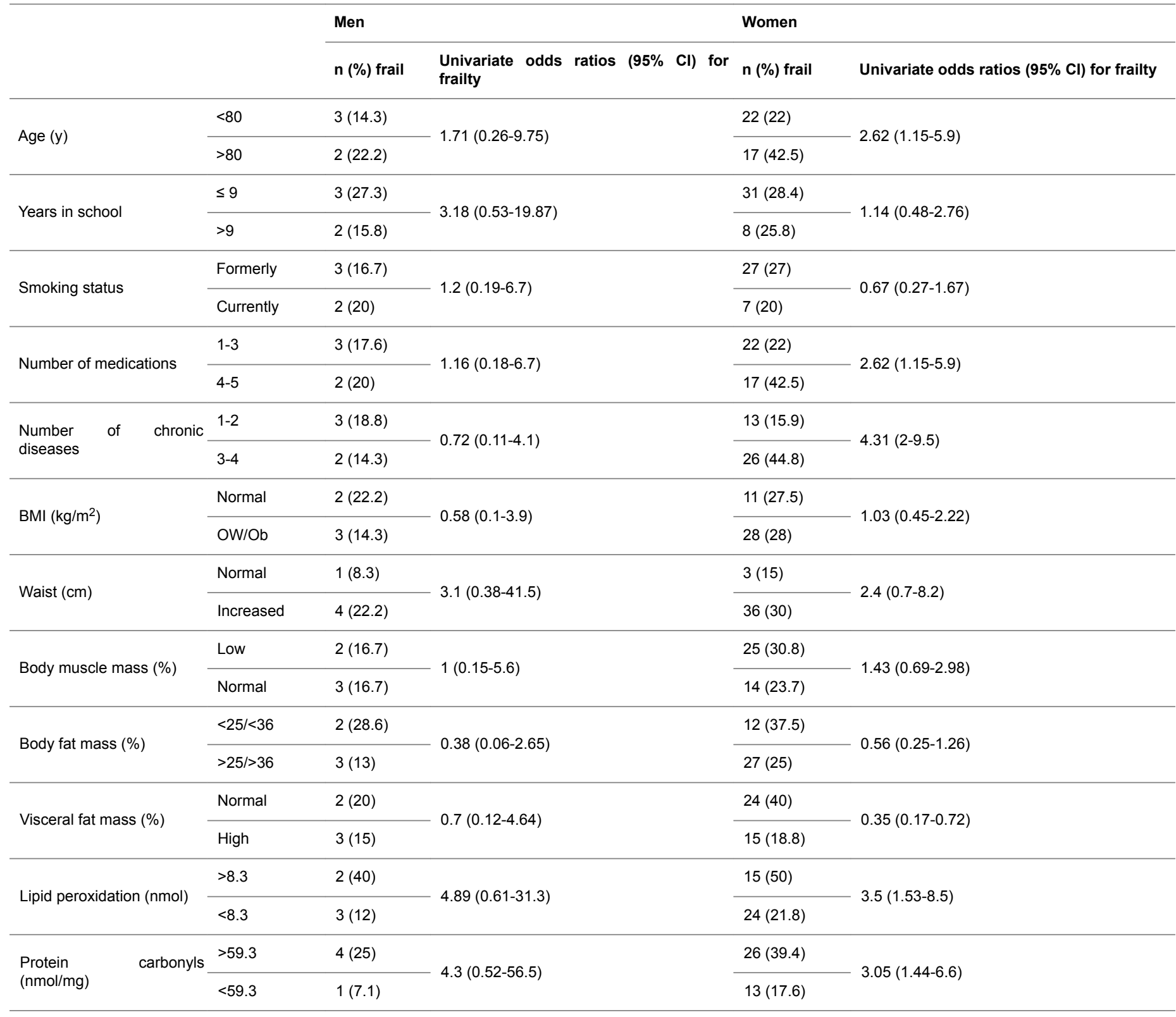

The association of physical performance battery tests and quality of life with frailty is shown in Table 3 . Men showed increased odds of frailty with low performance in the 6-min walk (OR 1.83, 95\% CI 0.11-15.1), the 2-min step test (OR 1.63, 95\% CI 0.29-10.2) and mobility (OR 2.25, 95\% CI 0.27-30), usual activities (OR 3.7, 95\% CI 0.45-48), pain (OR $10.3,95 \%$ CI $1.17-131$ ) and quality of life (OR $4.75,95 \%$ CI $0.77-29.8$ ). Women showed increased odds of frailty with low performance in the 2-min step test (OR 3.15, 95\% CI 1.5-6.5), the sit-up test (OR 11.2, 95\% CI 3.1-39.7), the handgrip strength test (OR 3.36, 95\% CI 1.6-7.1) and the items of mobility (OR 2.7, 95\% CI 1.3-5.7), usual activities (OR 2.88, 95\% CI 1.28-6.4), pain (OR 7.2, 95\% CI 3.2-15.4), anxiety (OR 3.5, 95\% CI 1.4-8) and quality of life (OR 2.4, 95\% CI 1.15-5.2). 
Association of physical performance tests with frailty indicators and oxidative stress markers in a sample of a community-dwelling elderly population

Table 3. Univariate associations between frailty and physical performance health related quality of life characteristics and oxidative markers.

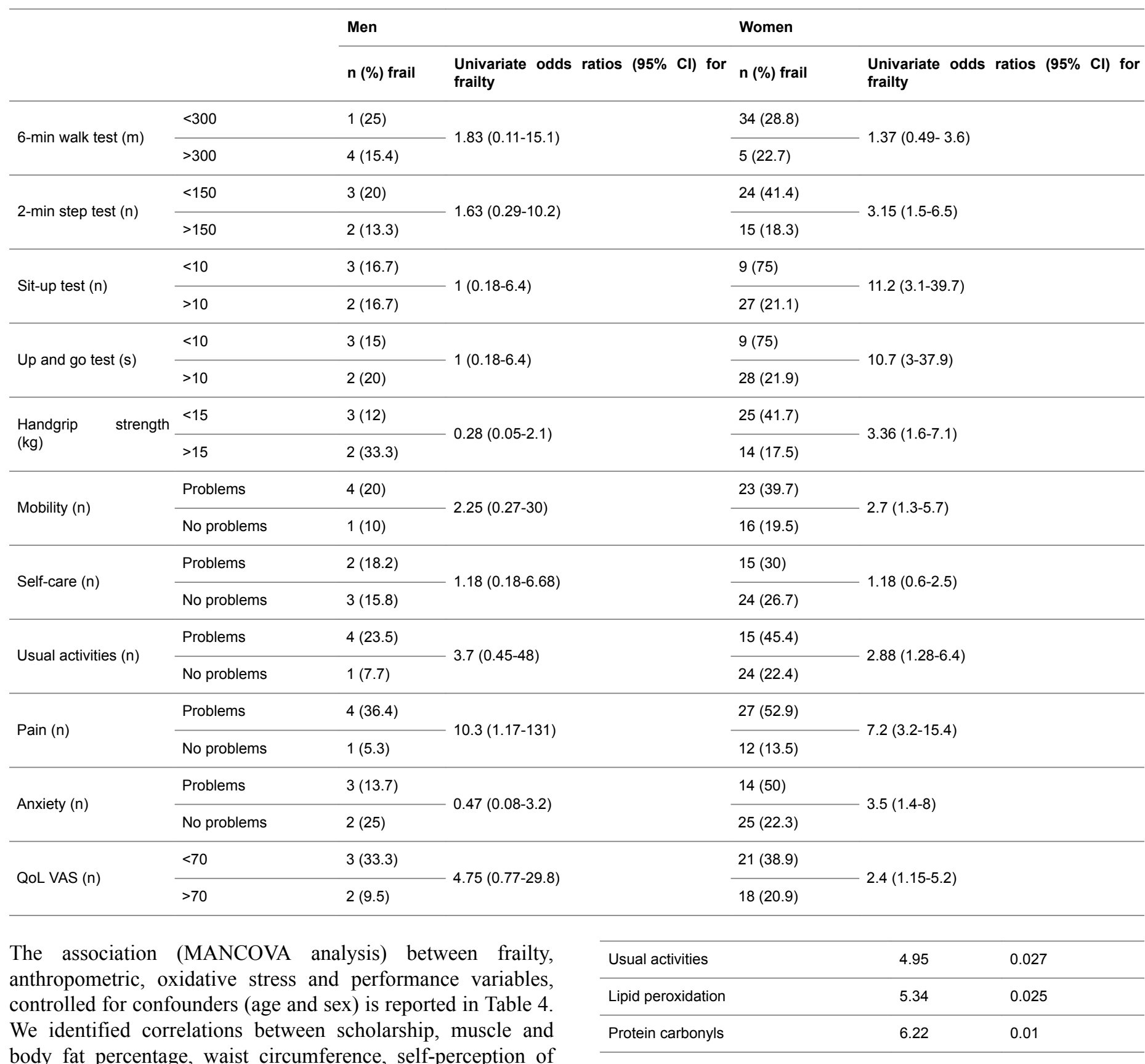
mobility and usual activities as well as with elevated lipid peroxidation and protein carbonylation levels.

Table 4. Correlation (MANCOVA) between frailty and related factors adjusted for age and sex.

\begin{tabular}{lll}
\hline & $\mathbf{F}$ & $\mathbf{p}$ \\
\hline Scholarship & 11.22 & 0.001 \\
\hline Comorbidities & 6.38 & 0.01 \\
\hline Waist circumference & 6.31 & 0.01 \\
\hline Muscle mass & 5.16 & 0.024 \\
\hline Total fat mass & 9.17 & 0.003 \\
\hline Mobility & 19.57 & $<0.0001$ \\
\hline
\end{tabular}

\section{Discussion}

This study is to our knowledge, the first to simultaneously assess the influence of body composition, social, clinical, quality of life, metabolic and oxidative stress parameters and physical performance in a community-dwelling elderly population. Results obtained pose the possibility of associate biomarkers of oxidative stress with physical tests to identify early stages of frailty in the elderly.

The frequency of frailty in this sample was $25.9 \%$ in persons over $65 \mathrm{y}$, which is consistent with other previous studies in Mexico [6] and in larger studies developed in other countries [5]. In our study, frailty was more frequent in women $(27.9 \%$ women $v$ s. $16.7 \%$ men). This gender difference is accentuated in women over the age of $80 \mathrm{y}$. 
As anticipated, the frequency of frailty in this study increased with age. Persons $>80 \mathrm{y}$ increase their odd of frailty, resulting in twice the incidence $v s$. those aged $<80 \mathrm{y}$.

On the other hand, previous studies have reported that walking speed is predictive of frailty and adverse outcomes [17] and that a poor performance in the 6 and 2 min tests increases the odds risk of frailty [18]. In this study we detected an association between both; the sit up test and the up and go test with frailty.

It has been reported an association between frailty and markers of inflammation and oxidative stress [19]. Carbonylation is associated with the accumulation of advanced glycation endproducts (AGEs) [20], which can activate the proinflammatory AGE receptors (RAGE)

We report here that indicators of oxidative stress (protein carbonylation and lipid peroxidation) significantly relate with frailty. Oxidative stress can activate cell apoptotic pathways and promote a decline in mitochondrial bioenergetics amongst many other detrimental effects [12]. The activation of these events by oxidative stress may explain detrimental effects on skeletal muscle that leads to loss of mass and strength, reducing functional independence and thereby promoting frailty [21]. Indeed, increased protein carbonylation has been associated with decreased handgrip strength [22]. Thus, a redox imbalance due to ageing can accentuate a decrease in muscle strength and mobility leading to the further development of weakness and slowness [23].

A possible link between oxidative stress and frailty was previously evaluated and the results failed to evidence a clear association [24]. In contrast our findings suggest that increased levels of protein carbonylation and lipid peroxidation associate to low physical performance as assessed by the battery of frailty tests used, in concordance with self-reported low physical performance as well as muscle and fat mass imbalance.

Our results are in agreement with the suggestion of the association oxidative stress and inflammation as pathways to frailty [25] and with the reported results of the ESTHER Cohort Study [26] suggesting and association of oxidative stress and CRP in the development of frailty, with reports suggesting association of altered redox balance [27], oxidative stress and cognitive decline [28] and frailty [28,29]. Oxidative stress also is related to frailty in subjects with Alzheimer disease suggesting a broader effect in the pathologies related to these alterations in the elderly [30].

\section{Conclusion}

Results reported herein evidence a strong association among physical performance, quality of life, metabolic and oxidative stress parameters in a community-dwelling elderly population. These results can be the base for future implementation of interventional studies to decrease frailty in the elderly with an integrated approach.

\section{Acknowledgment}

\section{Funding}

CONACyT 253769 to GC and NIHDK98717 \& AG47326 to FV; FV and GC are stockholders of Cardero Therapeutics; all rest authors declare no interest conflict. Funding was used only to research purposes.

\section{References}

1. Instituto Nacional De Estadistica Y Geografia. Censo de Población y Vivienda. INEGI 2010.

2. Sousa AS, Guerra RS, Fonseca I, Piche F, Ferreira S, Amaral TF. Financial impact of sarcopenia on hospitalization costs. Eur J Clin Nutr 2016; 70: 1046-1051.

3. Clegg A, Young J, Iliffe S, Rikkert MO, Rockwood K. Frailty in elderly people. The Lancet 2013; 381: 752-762.

4. Fried LP, Tangen CM, Walston J. Cardiovascular health study collaborative research group. Frailty in older adults: evidence for a phenotype. J Gerontol A Biol Sci Med Sci 2001; 56: 146-156.

5. Da Mata FAF, Pereira PP, de Andrade KRC, Figuereido AC, Silva MT, Pereira MG. Prevalence of frailty in Latin America and the caribbean: A systematic review and meta-analysis. PLoS One 2016; 8: e0160019.

6. García-Peña C, Ávila-Funes JA, Dent E, GutierrezRobledo L, Perez-Zepeda M. Frailty prevalence and associated factors in the Mexican health and aging study: A comparison of the frailty index and the phenotype. Exp Gerontol 2016; 79: 55-60.

7. Rantanen T, Avlund K, Suominen H, Schroll M, Frandin $\mathrm{K}$, Pertti E. Muscle strength as a predictor of onset of ADL dependence in people aged 75 years. Aging Clin Exp Res 2002; 3: 10-15.

8. Onder G, Penninx BW, Ferrucci L, Fried LP, Guralnik JM, Pahor M. Measures of physical performance and risk for progressive and catastrophic disability: results from the Women's Health and Aging Study. J Gerontol A Biol Sci Med Sci 2005; 1: 74-79.

9. Al Snih S, Markides KS, Ottenbacher KJ, Raji MA. Hand grip strength and incident ADL disability in elderly Mexican Americans over a seven-year period. Aging Clin Exp Res 2004; 6: 481-486.

10. Manrique-Espinoza B, Salinas-Rodríguez A, MorenoTamayo. Condiciones de salud y estado funcional de los adultos mayores en México. Salud Pública Mex 2013; 2: S323-S331.

11. Manrique-Espinoza B, Salinas-Rodríguez A, Salgado de Snyder N, Gutierrez-Robledo LM, Avila-Funes JA. Frailty and social vulnerability in Mexican deprived and rural settings. J Aging Health 2015; 4: 740-752.

12. Soysal P, Isik AT, Carvalho AF. Oxidative stress and frailty: A systematic review and synthesis of the best evidence. Maturitas 2017; 99: 66-72. 
13. Moreira PL, Villas Boas PJ, Ferreira AL. Association between oxidative stress and nutritional status in the elderly. Revista da Associacao Medica Brasileira 2014; 1 : 75-83.

14. Onat A, Can G, Kaya H, Hergenç G. Atherogenic index of plasma $(\log 10$ triglyceride/high-density lipoproteincholesterol) predicts high blood pressure, diabetes, and vascular events. J Clin Lipidol 2010; 2: 89-98.

15. Giannini C, Santoro N, Caprio S. The triglyceride-to-HDL cholesterol ratio. Association with insulin resistance in obese youths of different ethnic backgrounds. Diabetes Care 2011; 34: 1869-1874.

16. Nord E. EuroQol: health-related quality of life measurement. Valuations of health states by the general public in Norway. Health Policy 1991; 18: 25-36.

17. Castell M-V, Sánchez M, Julián R, Queipo R, Martin S, Otero A. Frailty prevalence and slow walking speed in persons age 65 and older: implications for primary care. BMC Family Practice 2013; 14: 86.

18. Millor N, Lecumberri P, Gómez M, Martinez-Ramirez A, Izquuierdo M. An evaluation of the 30 -s chair stand test in older adults: frailty detection based on kinematic parameters from a single inertial unit. J Neuroeng Rehabil 2013; $10: 86$.

19. Liu CK, Lyass A, Larson MG. Biomarkers of oxidative stress are associated with frailty: the Framingham Offspring Study. Age (Dord) 2016; 38: 1.

20. Hubbard RE, O'Mahony MS, Savva GM, Calver BL, Woodhouse KW. Inflammation and frailty measures in older people. J Cell Mol Med 2009; 13: 3103-3109.

21. Payne BAI, Chinnery PF. Mitochondrial dysfunction in aging: Much progress but many unresolved questions. Biochim Biophys Acta 2015; 1847: 1347-1353.

22. Howard C, Ferrucci L, Sun K. Oxidative protein damage is associated with poor grip strength among older women living in the community. J Appl Physiol 2007; 103: 17-20.

23. Pierre N, Appriou Z, Gratas-Delamarche A, Derbre F. From physical inactivity to immobilization: Dissecting the role of oxidative stress in skeletal muscle insulin resistance and atrophy. Free Rad Biol Med 2016; 98: 197-207.

24. Ingles $\mathrm{M}$, Gambini $\mathrm{J}$, Carnicero JA. Oxidative stress is related to frailty, not to age or sex, in a geriatric population: lipid and protein oxidation as biomarkers of frailty. J Am Geriatrics Soc 2014; 62: 1324-1328.

25. Ershler WB. A gripping reality: oxidative stress, inflammation, and the pathway to frailty. J Appl Physiol 2007; 103: 3-5.

26. Saum KU, Dieffenbach AK, Jansen EH. Association between oxidative stress and frailty in an elderly German population: Results from the ESTHER cohort study. Gerontology 2015; 61: 407-415.

27. Serviddio G, Romano AD, Greco A. Frailty syndrome is associated with altered circulating redox balance and increased markers of oxidative stress. Int J Immunopathol Pharmacol 2009; 22: 819-827.

28. Mulero J, Zafrilla P, Martinez-Cacha A. Oxidative stress, frailty and cognitive decline. J Nutr Health Aging 2011; 15: 756-760.

29. Wu IC, Shiesh SC, Kuo PH, Lin XZ. High oxidative stress is correlated with frailty in elderly Chinese. J Am Geriatr Soc 2009; 57: 1666-1671.

30. Namioka N, Hanyu H, Hirose D, Hatanaka H, Sato T, Shimizu S. Oxidative stress and inflammation are associated with physical frailty in patients with Alzheimer's disease. Geriatr Gerontol Int 2017; 17: 913-918.

\section{*Correspondence to}

Nayelli Najera

Escuela Superior de Medicina

Instituto Politecnico Nacional

Mexico 